\title{
Dark Energy
}

\author{
Ranku Kalita \\ Guwahati, India \\ Email: rankukalita@gmail.com
}

Received 20 May 2015; accepted 27 June 2015; published 30 June 2015

Copyright (C) 2015 by author and Scientific Research Publishing Inc.

This work is licensed under the Creative Commons Attribution International License (CC BY).

http://creativecommons.org/licenses/by/4.0/

c) (i) Open Access

\begin{abstract}
It is proposed that the principle of equivalence-which recognizes momentary equivalence between gravitational and inertial force-is cosmologicalized in an expanding spherical distribution of matter, whereby spacetime itself acts as the accelerating frame of reference of the inertial mass of an object on the surface of the sphere, and the inertial force thus generated is momentarily equivalent at a certain radial distance to the gravitational force experienced by the gravitational mass of the object. The spacetime accelerating frame of reference is parametrized by the cosmological constant $\Lambda$. The relation between inertial mass of an object and the cosmological constant can be generalized as a correlation between the matter density in the universe and the cosmological constant. Since matter density dilutes in an expanding universe, it is predicted that the $\Lambda$-term should be time varying.
\end{abstract}

\section{Keywords}

Cosmology, Dark Energy, Dark Matter

\section{Introduction}

Dark energy which is accelerating the expansion of the universe presently constitutes about $69 \%$ of the universe [1]-[3]. The standard model mathematically parametrizes dark energy in the form of the cosmological constant $\Lambda$, where we have $\rho_{\Lambda} \propto a^{0}$ while $\rho_{M} \propto a^{-3}$ ( $\rho_{\Lambda}$ is mass density of cosmological constant $\Lambda$, as in $\rho_{\Lambda}=\frac{\Lambda c^{2}}{8 \pi G}$, $a$ is expansion or scale factor, and $\rho_{M}$ is matter density). The accelerating expansion of the universe becomes evident at late times when $\Omega_{\Lambda}>\frac{1}{2} \Omega_{M}$, which occurred $\sim 6 \times 10^{9}$ years ago. The quest then is to physically explain the existence of the cosmological constant. 


\section{Dark Energy}

The accelerating recession between two objects is parametrized by the cosmological constant $\Lambda$ in the form of $\frac{\Lambda \mathrm{c}^{2}}{3} R$, which belongs to the equation of motion of an object on the surface of an expanding spherical distribution of matter, and is derived from the second Friedmann equation [4] [5]:

$$
\frac{\ddot{a}}{a}=-\frac{4 \pi G}{3}\left(\rho+3 P / c^{2}\right)+\frac{\Lambda c^{2}}{3},
$$

which may be re-framed as the equation of motion of mass $m$ on the surface of a sphere of radius $R \equiv a$ and mass $M$

$$
\begin{gathered}
\ddot{R}=-\frac{G M}{R^{2}}+\frac{\Lambda c^{2}}{3} R . \\
{\left[\text { Total “gravitating mass” } M=\frac{4 \pi}{3} R^{3}\left(\rho+3 P / c^{2}\right)\right]}
\end{gathered}
$$

If the spherical distribution of matter is expanding, there will be a moment of equilibrium between the gravitational force and the antigravitational force on the object $m$ on the surface of the sphere at a certain radial distance. The gravitational force acts on the gravitational mass of the object. Now what might the antigravitational force be acting upon the object? The gravitational force exists by virtue of the existence of the gravitational mass of the object. Does the antigravitational force also exist by virtue of the existence of mass of the object? But the gravitational mass of the object is already utilized. What other property of mass may be available?

We know that the mass of an object can be dually described as gravitational and inertial mass, and remarkably enough they are also equivalent. Might the antigravitational force be acting upon the inertial mass of the object?

If the antigravitational force is acting upon the inertial mass of the object, then that would generate inertial force, whose direction would be the same as the gravitational force acting upon the object, and would be directed toward the center of the spherical distribution of matter. Thus at the moment of equilibrium between the gravitational force and antigravitational force there would also be momentary equivalence between the gravitational force and inertial force upon the object. It is proposed that this constitutes the cosmologicalization of the principle of equivalence, which recognizes momentary equivalence between: 1) the gravitational force experienced by the gravitational mass of an object situated on a gravitational surface, and 2) the inertial force generated on the inertial mass of an object in a uniformly accelerating frame of reference (see also Appendix).

In the principle of equivalence, the frame of reference of the inertial mass of an object exists with respect to the background of spacetime, and whose acceleration has to be imparted by an external force; while in the cosmologicalization of the principle of equivalence, it is spacetime itself that is the frame of reference of the inertial mass of an object, and whose the acceleration is parametrized by $\frac{\Lambda c^{2}}{3} R$.

The acceleration of the frame of reference of the inertial mass of an object is uniform in the principle of equivalence; while in the cosmologicalization, the acceleration is non-uniform parametrized by $\frac{\Lambda c^{2}}{3} R$.

In the principle of equivalence the momentary equivalence between gravitational and inertial force has to be demonstrated by considering the object in two distinct frames of reference-one frame of reference involves the static gravitational mass of an object, as in 1), while the other frame of reference involves the dynamic inertial mass of an object, as in 2); in the cosmologicalization, the equivalence between gravitational and the inertial force occurs in the spacetime frame of reference of the same dynamic object on the surface of the expanding sphere $^{1}$.

As mentioned, the cosmological constant parametrizes the spacetime accelerating frame of reference of the inertial mass of an object. The relation between the cosmological constant and the inertial mass of an object may

\footnotetext{
${ }^{1}$ The cosmologicalization of the principle of equivalence may also offer a classical mechanical explanation about the nature of dark matter as well [6]; the paper by the present author also discusses the nature of dark energy in a similar vein, but lacked a prediction, which the present paper offers.
} 
be generalized as a correlation between the matter density in the universe and the cosmological constant by considering the second Friedmann equation (Equation (1)) for a static universe.

In a static universe, $\ddot{a}=0$, and assuming a pressureless universe, we have

$$
\frac{\Lambda c^{2}}{3}-\frac{4 \pi G \rho}{3}=0
$$

or,

$$
\Lambda c^{2}=4 \pi G \rho .
$$

Here $\rho$ represents gravitational mass (which for the present proposition shall represent only matter), and which is counterrelated to the antigravitational term $\Lambda$ in Equation (3). However, it will be noted that in Equation (4) the gravitational and the antigravitational terms are correlated, which is physically contradictory. This can be resolved, however, by recalling the equivalence of gravitational and inertial mass, and interpreting $\rho$ in equation (4) in terms of inertial mass, and thereby rendering the correlation as between inertial mass and the cosmological constant ${ }^{2}$.

The correlation between $\rho$ and $\Lambda$ derived for a static universe from the second Friedmann equation cannot however be mathematically extrapolated to a dynamic universe, since $\ddot{a} \neq 0$ in Equation (1) for such a universe.

Let us then turn to the first Friedmann equation, but first without the cosmological constant:

$$
H^{2} \equiv\left(\frac{\dot{a}}{a}\right)^{2}=\frac{8 \pi G \rho}{3}-\frac{k c^{2}}{a^{2}},
$$

where $\rho$ in the term $\frac{8 \pi G \rho}{3}$ represents matter and radiation density in the universe. We know that the Hubble parameter $H$ has to decrease over time, since it is inversely proportional to the increasing age of the universe; and this is consistent with the fact that $\rho$ in $\frac{8 \pi G \rho}{3}$ also decreases in an expanding universe.

Let us now consider the Friedmann equation with the cosmological constant:

$$
H^{2} \equiv\left(\frac{\dot{a}}{a}\right)^{2}=\frac{8 \pi G \rho}{3}-\frac{k c^{2}}{a^{2}}+\frac{\Lambda c^{2}}{3} .
$$

Here, the physical meaning of $\rho$ has changed: $\frac{8 \pi G \rho}{3}$ now represents dark energy, which can be derived from the gravitational term $-\frac{4 \pi G}{3}\left(\rho+3 P / c^{2}\right)$ in the second Friedmann equation (Equation (1))-since the cosmological constant can be considered as an ideal fluid with negative pressure, whereby $p / c^{2}=-\rho$.

Now the prevailing practice has been to consider $\rho$ in Equation (6) as encompassing dark energy, matter and radiation. Since the Hubble parameter $H$ has to decrease over time in Equation (6) for the age of the universe to increase, it is sufficient for matter and radiation density to decrease while dark energy density can remain constant. Indeed, so far the astronomical observational constraints are consistent with a time-independent cosmological constant. However, it may be argued that since $\frac{8 \pi G \rho}{3}$ already includes matter and radiation in Equation (5), while $\frac{8 \pi G \rho}{3}$ in Equation (6) can be explicitly derived from Equation (1) to signify dark energy, so $\frac{8 \pi G \rho}{3}$ in Equation (6) should exclusively represent dark energy. The exclusivity of $\frac{8 \pi G \rho}{3}$ to represent dark energy in Equation (6) is further strengthened when we consider that negative pressure, dark energy density, and the cosmological constant correspond as

\footnotetext{
${ }^{2}$ In the static universe model (Equation (3)), it will be noted that for different initial values of $\rho$ the value of $\Lambda$ would have to vary as wellthus, $\Lambda$ is not an independent fundamental constant.
} 


$$
p=\rho=\frac{\Lambda c^{2}}{8 \pi G} .
$$

But we can also get $\rho=\frac{\Lambda c^{2}}{8 \pi G}$ from Equation (6) by setting

$$
\frac{8 \pi G \rho}{3}=\frac{\Lambda c^{2}}{3}
$$

Thus $\frac{8 \pi G \rho}{3}$ should exclusively represent dark energy in Equation (6). And since $H$ has to decrease in Equation (6) for the age of the universe to increase, $\rho$ in $\frac{8 \pi G \rho}{3}$ must also decrease.

Though the present astronomical observational constraints are that of a time-independent cosmological constant, the error bars are large enough to allow for the possibility of the detection of a slowly time-varying cosmological constant either in ongoing or future astronomical observations. It is however not possible to predict the constraints on a time-varying $\Lambda$-term from the Friedmann equation itself-that may only be empirically determined from astronomical observations ${ }^{3}$.

The curious coincidence of the term $\frac{8 \pi G \rho}{3}$ occurring in both the Equations (5) and (6), in each of which $\rho$ represents distinct types of contents of the universe, is noteworthy. It may be argued that this should not be seen merely as a coincidence but can also be seen as implying a correlation between diluting matter density (for the present proposition only matter density is considered) in Equation (5) and dark energy density in Equation (6), if dark energy density were to also decrease in an expanding universe.

\section{Conclusion}

As proposed, a correlation between matter density and the cosmological constant would imply a correlation between the inertial mass of matter in the universe and the cosmological constant, which in turn would be a generalized expression of the cosmologicalization of the principle of equivalence in an expanding spherical distribution of matter, whereby the inertial mass of an object on the surface of the sphere has to exist in a spacetime accelerating frame of reference, which is responsible for the accelerating expansion of the universe.

\section{References}

[1] Adam, R., Ade, P.A.R., Aghanim, N., Akrami, Y., Alves, M.I.R., et al. (2015) Planck 2015 Results. I. Overview of Products and Scientific Results. arXiv:1502.01582v1 [astro-ph.CO].

[2] Riess, A.G., Filippenko, A.V., Challis, P., Clocchiatti, A., Diercks, A., et al. (1998) Astrophysical Journal, 116, 10091038. http://dx.doi.org/10.1086/300499

[3] Perlmutter, S., Aldering, G., Goldhaber, G., Knop, R.A., Nugent, P., et al. (1999) Astrophysical Journal, 517, 565-586. http://dx.doi.org/10.1086/307221

[4] Friedmann, A. (1922) Zeitschrift für Physik, 10, 377-386.

Friedmann, A. (1999) General Relativity and Gravitation, 31, 1991-2000. http://dx.doi.org/10.1007/BF01332580

[5] Friedmann, A. (1924) Zeitschrift für Physik, 21, 326-332.

Friedmann, A. (1999) General Relativity and Gravitation, 31, 2001-2008. http://dx.doi.org/10.1023/A:1026755309811

[6] Kalita, R. (2014) International Journal of Astronomy, 3, 18-21. http://dx.doi.org/10.5923/j.astronomy.20140301.02

[7] Peebles, P.J.E. and Ratra, B. (1988) Astrophysical Journal, 325, L17-L20. http://dx.doi.org/10.1086/185100

[8] Sahni, V. and Starobinsky, A. (2000) International Journal of Modern Physics D, 9, 373-443. http://dx.doi.org/10.1142/S0218271800000542

${ }^{3}$ The possibility of a time-varying $\Lambda$-term has already been proposed in scalar field models, but they offer no fundamental physical rationale as to why the $\Lambda$-term should be time varying, beyond the formulation that that the scalar field's "stress-energy tensor acts like a time-variable cosmological constant" [7]. In the present proposition, however, a fundamental physical rationale for a time-varying $\Lambda$-term is offeredin terms of a correlation between diluting matter density and the cosmological constant, which is an expression of the proposed cosmologicalization of the principle of equivalence. 
[9] Einstein, A. (1917) Sitzungsberichte der Preussischen Akademie der Wissenschaften, Berlin, 142-152.

[10] Mach, E. (1919) The Principles of Dynamics. In: Mach, E., The Science of Mechanics, Open Court, Chicago, 236.

\section{Appendix}

One of the early aims in general relativity was to incorporate the Mach's principle [8]. Ernst Mach contested the Newtonian notion of primacy of local description of the motion of inertial mass. Mach held that the local description of the motion of an inertial mass does not suffice, nor does referencing it to the background of absolute space-he argued instead that the description of the motion of an inertial mass is only physically meaningful relative to any other mass. When Einstein developed the general theory of relativity he agreed with Mach: "In a consistent theory of relativity there can be no inertia relatively to "space”, but only an inertia of masses relatively to one another" [9].

Einstein introduced the cosmological constant for the purpose of constructing a model of a static universe, and which was also expected to incorporate the Mach's principle. Such a universe was however shown to be unstable, and with Hubble's discovery that the universe is expanding, the purpose of introducing the cosmological constant was rendered redundant. In 1998 the discovery of the accelerating expansion of the universe was announced, and the cosmological constant had to be resurrected to describe the dynamics of such a universe. Thus we may therefore also revisit as to how the cosmological constant may enable the incorporation of the Mach's principle, this time in a dynamic universe.

There is a deeper philosophical motivation to the Mach's principle, which is that the proposition that the motion of the inertial mass of an object is only physically meaningful relative to any other (inertial) mass in the universe, puts the inertial mass of an object on a par with the equivalent gravitational mass of the object, whose motion-due to the interacting gravitational force-requires the existence of at least another gravitational mass; in other words, the motion of a gravitational mass is only physically meaningful relative to any other gravitational mass in the universe. As Mach stated: "It is not necessary to refer the law of inertia to a special absolute space. On the contrary, it is perceived that the masses that in the common phraseology exert forces on each other as well as those that exert none, stand with respect to acceleration in quite similar relations. We may, indeed, regard all masses as related to each other.” [10].

The present proposition of the cosmologicalization of the principle of equivalence identifies the antigravitational force responsible for the presently accelerating expansion of the universe as involving the inertial mass of an object $m$ on the surface of an expanding spherical distribution of matter $M$, whereby it is spacetime itself which acts as an accelerating frame of reference of the object $m$, and thereby generates inertial force upon the inertial mass of the object $m$, and which would be momentarily equivalent at a certain radial distance to the gravitational force upon the object $m$. It will be noted that $\frac{\Lambda c^{2}}{3} R$ in Equation (2) which describes the antigravitational acceleration upon the object $m$ is also describing the motion of the object $m$ relative to the object $M$ in terms of the distance $R$ between the center of mass of the objects $m$ and $M$; and, $-\frac{G M}{R^{2}}$ in Equation (2) which describes the gravitational acceleration upon the object $m$ is also describing the motion of the object $m$ relative to the object $M$ in terms of the distance $R^{2}$ between the center of mass of the objects $m$ and $M$. This is precisely what the Mach's principle proposes: that the motion of the inertial mass of an object is only physically meaningful relative to any other mass, just as the motion of the gravitational mass of an object is only physically meaningful relative to any other mass. Thus it may be argued that the present proposition of the cosmologicalization of the principle of equivalence to explain the antigravitational force accelerating the expansion of the universe also accomplishes the long-held goal of incorporating the Mach's principle into general relativity. 